Mindnich, Anja; Berger, Stefanie; Fritsch, Sabine

\title{
Modellierung des fachlichen und fachdidaktischen Wissens von Lehrkräften im Rechnungswesen - Überlegungen zur Konstruktion eines
}

\section{Testinstruments}

Faßhauer, Uwe [Hrsg.]; Fürstenau, Bärbel [Hrsg.]; Wuttke, Eveline [Hrsg.]: Jahrbuch der berufs- und wirtschaftspädagogischen Forschung 2013. Opladen [u.a.] : Verlag Barbara Budrich 2013, S. 61-72. (Schriftenreihe der Sektion Berufs- und Wirtschaftspädagogik der Deutschen Gesellschaft für Erziehungswissenschaft (DGfE))

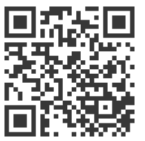

Quellenangabe/ Reference:

Mindnich, Anja; Berger, Stefanie; Fritsch, Sabine: Modellierung des fachlichen und fachdidaktischen Wissens von Lehrkräften im Rechnungswesen - Überlegungen zur Konstruktion eines Testinstruments - In: Faßhauer, Uwe [Hrsg.]; Fürstenau, Bärbel [Hrsg.]; Wuttke, Eveline [Hrsg.]: Jahrbuch der berufs- und wirtschaftspädagogischen Forschung 2013. Opladen [u.a.] : Verlag Barbara Budrich 2013, S. 61-72 - URN: urn:nbn:de:0111-opus-80648 - DOI: 10.25656/01:8064

https://nbn-resolving.org/urn:nbn:de:0111-opus-80648

https://doi.org/10.25656/01:8064

in Kooperation mit / in cooperation with:

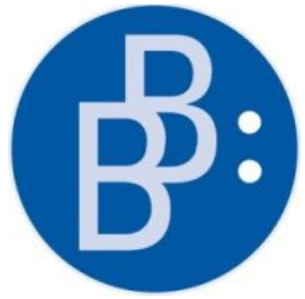

https://www.budrich.de

\section{Nutzungsbedingungen}

Dieses Dokument steht unter folgender Creative Commons-Lizenz: http://creativecommons.org/licenses/by-nc-nd/3.0/de/deed - Sie dürfen das Werk bzw. den Inhalt unter folgenden Bedingungen vervielfältigen, verbreiten und öffentlich zugänglich machen: Sie müssen den Namen des Autors/Rechteinhabers in der von ihm festgelegten Weise nennen. Dieses Werk bzw. dieser Inhalt darf nicht für kommerzielle Zwecke verwendet werden und es darf nicht bearbeitet, abgewandelt oder in anderer Weise verändert werden.

Mit der Verwendung dieses Dokuments erkennen Sie die Nutzungsbedingungen an.

\section{Terms of use}

This document is published under following Creative Commons-License: http://creativecommons.org/licenses/by-nc-nd/3.0/de/deed.en - You may copy, distribute and transmit, adapt or exhibit the work in the public as long as you attribute the work in the manner specified by the author or licensor. You are not allowed to make commercial use of the work or its contents. You are not allowed to alter, transform, or change this work in any other way.

By using this particular document, you accept the above-stated conditions of use.

\section{Kontakt / Contact:}

peDOCS

DIPF | Leibniz-Institut für Bildungsforschung und Bildungsinformation Informationszentrum (IZ) Bildung

E-Mail: pedocs@dipf.de

Internet: www.pedocs.de

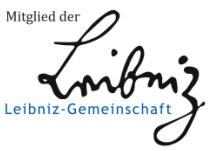




\section{Jahrbuch der berufs- und wirtschaftspädagogischen Forschung 2013}

Uwe Faßhauer, Bärbel Fürstenau, Eveline Wuttke (Hrsg.)

DGfE Deutsche Gesellschaft 
Jahrbuch der berufs- und wirtschaftspädagogischen Forschung 2013 
Schriftenreihe der Sektion

Berufs- und Wirtschaftspädagogik der Deutschen Gesellschaft

für Erziehungswissenschaft (DGfE) 
Uwe Faßhauer

Bärbel Fürstenau

Eveline Wuttke (Hrsg.)

Jahrbuch der berufs- und

wirtschaftspädagogischen

Forschung 2013

Verlag Barbara Budrich

Opladen • Berlin • Toronto 2013 
Bibliografische Information der Deutschen Nationalbibliothek

Die Deutsche Nationalbibliothek verzeichnet diese Publikation in der Deutschen Nationalbibliografie; detaillierte bibliografische Daten sind im Internet über http://dnb.d-nb.de abrufbar.

(C) Dieses Werk ist im Verlag Barbara Budrich erschienen und steht unter folgender Creative Commons Lizenz: http://creativecommons.org/licenses/by-nc-nd/3.0/de/ Verbreitung, Speicherung und Vervielfältigung erlaubt, kommerzielle Nutzung und Veränderung nur mit Genehmigung des Verlags Barbara Budrich.

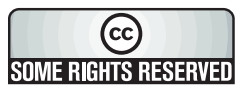

Dieses Buch steht im OpenAccess Bereich der Verlagsseite zum kostenlosen Download bereit (http://dx.doi.org/10.3224/978384740127)

Eine kostenpflichtige Druckversion (Printing on Demand) kann über den Verlag bezogen werden. Die Seitenzahlen in der Druck- und Onlineversion sind identisch.

\section{ISBN 978-3-8474-0127-8 \\ DOI $\quad 10.3224 / 978384740127$}

Umschlaggestaltung: bettina lehfeldt graphic design, Kleinmachnow Typografisches Lektorat: Ulrike Weingärtner, Gründau Verlag Barbara Budrich, http://www.budrich-verlag.de 


\title{
Teil I: Lehr-Lernforschung in der beruflichen Bildung
}

\author{
Jan Hendrik Stork \\ Der Einfluss unterschiedlicher Unterrichtsmodelle auf den Erwerb \\ von mathematischen und kaufmännischen Kompetenzen im
} beruflichen Unterricht

Mandy Hommel

„Darf ich um Ihre Aufmerksamkeit bitten?!” Wege aus dem

Aufmerksamkeitstief....

Stephan Schumann, Franz Eberle, Maren Oepke

Ökonomisches Wissen und Können am Ende der Sekundarstufe II:

Effekte der Bildungsgang-, Klassen- und Geschlechtszugehörigkeit

Christine Caroline Jähnig

Assessing Business Knowledge of Students in German

Higher Education

Anja Mindnich, Stefanie Berger, Sabine Fritsch

Modellierung des fachlichen und fachdidaktischen Wissens von

Lehrkräften im Rechnungswesen - Überlegungen zur Konstruktion

eines Testinstruments.

Roland Happ, Susanne Schmidt, Olga Zlatkin-Troitschanskaia

Der Stand des wirtschaftswissenschaftlichen Fachwissens von

Bachelorabsolventen der Universität und der Fachhochschule.

\section{Teil II: Didaktik und Methodik beruflichen Lernens}

Nicole Kimmelmann, Wilhelmine Berg

Wie viel Deutsch darf's sein? Das Projekt „Deutsch am Arbeitsplatz”

Claudia Stolp, Jens Siemon

Wirkung auf Lernerfolg und Motivation durch Debriefing in

Unternehmensplanspielen . 99 
Georg Tafner

Supranationalität begreifbar machen. Performative Pädagogik

im Planspiel.

Frank Arens

Praxisbegleitung in der Pflegeausbildung - ein blinder Fleck der

Berufsbildungsforschung?

Axel Grimm

Lehrerhandeln im computerunterstützten Berufsschulunterricht Handlungsmuster von Berufsschullehrkräften in elektro- und metalltechnischen Lehr-Lernarrangements

\section{Teil III: Hochschuldidaktik und Lehrerbildung}

\section{Gabriela Kugler}

Grundstrukturen professionellen Lehrerwissens: Wissen zur

Lesekompetenzförderung von Berufsschullehrkräften

Juliana Schlicht

Forschendes Lernen im Studium: Ein Ansatz zur Verknüpfung von

Forschungs-, Lehr- und Lernprozessen.

Romy Wolff

Interaktionsprozesse zur Entscheidungsfindung in virtuellen und face-to-face Gruppen

Ulrike Weyland, Karin Reiber

Lehrer/-innen-Bildung für die berufliche Fachrichtung Pflege in hochschuldidaktischer Perspektive

\section{Teil IV: Berufs- und wirtschaftspädagogische Perspektiven}

Wiebke Petersen

Prinzipien der Regulierung des Feldes der Berufsbildung - ein Ansatz zum internationalen Vergleich von „ValNIL” 203 
Stefan Wolf

Berufsbildung und Migration - kritische Anmerkungen über

vernachlässigte Perspektiven

Markus Linten, Christian Woll

Berufsbildungsdiskurs 2010 und 2011: Vergleichende Resonanzanalyse referierter und nicht-referierter Zeitschriftenbeiträge zur

Berufsbildungsforschung und -praxis

Martin Kröll

Das Zusammenspiel von Selbst- und Fremdorganisation in lernenden Organisationen

Gregor Thurnherr, Samuel Schönenberger \& Christian Brühwiler

Hilfreiche Unterstützung in der Berufsorientierung aus Sicht von

Jugendlichen 


\section{Modellierung des fachlichen und fachdidaktischen Wissens von Lehrkräften im Rechnungswesen - Überlegungen zur Konstruktion eines Testinstruments}

Anja Mindnich, Stefanie Berger \& Sabine Fritsch

\section{Erkenntnisinteresse und Forschungsvorhaben}

Die Kompetenzmessung im Hochschulbereich stellt bislang ein weitgehend vernachlässigtes Forschungsgebiet des Bildungssektors dar (vgl. Blömeke et al. 2013). Eine umfassende Analyse des internationalen Forschungsstandes im Bereich der Modellierung und Erfassung von Kompetenzen zeigt insbesondere für Europa ein Forschungsdefizit im Hochschulsektor auf (vgl. Kuhn/Zlatkin-Troitschanskaia 2011). Daher wurde 2010 vom BMBF das Forschungsprogramm „Kompetenzmodellierung und Kompetenzerfassung im Hochschulsektor" (KoKoHs) initiiert, welches die Ziele verfolgt, die Leistungsfähigkeit des tertiären Bildungssystems in Deutschland zu erhalten und Grundlagen für eine Evaluation der Kompetenzentwicklung sowie des Kompetenzerwerbs an Hochschulen zu schaffen (vgl. Blömeke et al. 2013, 3). Im Rahmen des Forschungsprogramms werden u.a. Verbundprojekte aus den Bereichen der Berufs- und Wirtschaftspädagogik (Projekt WiWiKom, siehe Zlatkin-Troitschanskaia/Breuer 2013) sowie der Lehrerbildung gefördert (für eine Übersicht der einzelnen Projekte siehe Blömeke/Zlatkin-Troitschanskaia 2013). Das Verbundprojekt „Modellierung und Erfassung fachwissenschaftlicher und fachdidaktischer Kompetenzen im wirtschaftspädagogischen Studium" (kurz KoMeWP: Kompetenzmessung im wirtschaftspädagogischen Studium; siehe Seifried/Wuttke/Schmitz 2011) verknüpft diese beiden Bereiche. ${ }^{1}$ Dabei wird die Zielsetzung verfolgt, die professionelle Kompetenz von angehenden Lehrkräften im kaufmännisch-verwaltenden Bereich zu modellieren und testtheoretisch abprüfbar zu machen.

Als Grundlage für die Modellierung der professionellen Kompetenz einer Lehrkraft dient in KoMeWP der Kompetenzbegriff sensu Weinert (2001). Demnach wird die professionelle Kompetenz als Leistungsdisposition aufgefasst, die sich als veränderbares Merkmal funktional auf Unterrichtssituationen des Rechnungswesens bezieht und sich durch ein Zusammenspiel

Das Verbundprojekt wird von der Forschergruppe um Seifried, Wuttke und Schmitz an den Universitäten in Mannheim (Förderkennzeichen: 01PK11003a), Frankfurt (Förderkennzeichen: 01PK11003b) und Darmstadt (Förderkennzeichen: 01PK11003c) bearbeitet. 
von professionellem Wissen, Überzeugungen, motivationalen Orientierungen und selbstregulativen Fähigkeiten charakterisieren lässt. ${ }^{2}$

Da das professionelle Wissen als erklärungsmächtigster Faktor von Expertenleistungen gilt (vgl. Gruber/Mandel 1996; Krauss et al. 2011), liegt der Fokus im Rahmen des Forschungsvorhabens auf der Erfassung dieses kognitiven Kompetenzaspektes - insbesondere auf der Erfassung des Fachwissens und des fachdidaktischen Wissens. Aktuell durchgeführte Studien zur professionellen Fehlerkompetenz (vgl. Seifried/Wuttke 2010; Seifried/ Türling/Wuttke 2010) zeigen, dass das fachwissenschaftliche und das fachdidaktische Wissen in der Ausbildung angehender Wirtschaftspädagogen nicht in hinreichendem Maße erworben werden.

Zur Konzeptualisierung des professionellen Wissens wird ein domänenspezifischer Zugang gewählt, um die Charakteristika des betrachteten Lerninhaltsbereichs sowohl aus fachlicher als auch aus fachdidaktischer Perspektive zu berücksichtigen. ${ }^{3}$ Im Rahmen des Projekts KoMeWP liegt der Fokus auf dem Lerninhaltsbereich Rechnungswesen, dem „Kern des betrieblichen Informationssystems" (Preiß 2000, 7). Da das Rechnungswesen als Grundlage für die „Überprüfung und Bewertung von betrieblichen Entscheidungsund Umsetzungsprozessen" dient, kommt ihm auch im Unterricht eine besondere Bedeutung zu, denn es fungiert hier als „Katalysator für eine allgemeine betriebswirtschaftliche Kompetenzerweiterung" seitens der Lernenden (Sloane 1996, 26).

Da bislang kein standardisiertes Testinstrument existiert, welches sowohl das fachliche als auch das fachdidaktische Wissen von Lehrkräften im Rechnungswesen erfasst, wird im KoMeWP-Projekt ein Paper-Pencil-Test konstruiert. Im Rahmen des vorliegenden Beitrags werden die Vorarbeiten zur Konstruktion des Testinstruments beschrieben und richtungsweisende Entscheidungen begründet. In einem ersten Schritt stellt sich die Frage, wie das professionelle Wissen theoretisch zu konzeptionieren ist (Kapitel 2). Außerdem gilt es zu klären, welche Rechnungsweseninhalte in den Testitems Beachtung finden sollen. Erforderlich ist in dieser Hinsicht eine Analyse des Inhaltsbereichs des (externen) Rechnungswesens (Kapitel 3). Abschließend werden die Vorarbeiten in einem Rahmenmodell für die Itemkonstruktion zusammengeführt (Kapitel 4).

2 Vergleichbare Konzeptualisierungen der Lehrerkompetenz werden auch in COACTIV (Kunter et al. 2011) oder TEDS-LT ( Blömeke et al. 2011) zugrunde gelegt.

3 Die im vorliegenden Artikel fokussierte Domäne ist bezogen auf den Rechnungswesenunterricht in kaufmännisch-verwaltenden beruflichen Schulen. Die Domäne wird zum einen begrenzt durch die Struktur der curricular verankerten Inhalte des externen Rechnungswesens und zum anderen durch die unterrichtsbezogenen Anforderungen in der praktischen Interaktion zwischen Lehrkraft und Lernenden (vgl. Baumert/Kunter 2011, 13). 


\section{Testtheoretische Konzeptualisierung des fachwissenschaftlichen und fachdidaktischen Wissens}

Die von Shulman (1986) vorgeschlagene Ausdifferenzierung des professionellen Wissens in die Komponenten Fachwissen (CK), fachdidaktisches Wissen (PCK) und allgemein-pädagogisches Wissen (PK) stellt aus heutiger Sicht eine allgemein akzeptierte Konzeptualisierung dar. Gleichzeitig bilden die Komponenten die Kernbereiche der Lehrerbildung in Deutschland ab, die durch Ausbildung und Unterrichtspraxis erworben werden (vgl. Tepner et al. 2012, 8f.). Die Bedeutung dieser „Kernkategorien” bei der Aufgabenbewältigung in Lehrberufen ist unbestritten (vgl. Krauss et al. 2011, 136). Da der vorliegende Aufsatz die konzeptionellen Grundlagen der Entwicklung eines domänenspezifischen, standardisierten Testinstruments zum Gegenstand hat, erfolgt nachstehend eine Einschränkung auf die beiden fachbezogenen Wissenskomponenten (CK und PCK).

\section{Fachwissen}

Gemäß Shulman $(1986,9)$ sollte eine Lehrkraft nicht nur über reines Faktenwissen verfügen, sondern insbesondere die Hintergründe und Struktur des Fachinhalts kennen. Das Rechnungswesen ist stark durch Modellstrukturen und -prozesse gekennzeichnet, die durch betriebswirtschaftliche Begriffe und Konzepte Eingang in die Fachstruktur finden. Im Rechnungswesenunterricht manifestieren sich demnach betriebswirtschaftliche Theorie und Praxis (vgl. Sloane 1996, 27). Dies erschwert eine strikte Grenzziehung beim Abstecken inhaltlicher Themengebiete. Für die Testkonstruktion ist es deshalb erforderlich, das rechnungswesenbezogene Fachwissen nicht nur inhaltlich näher zu spezifizieren (siehe Kapitel 3), sondern auch eine für (angehende) Lehrkräfte angemessene Niveaustufe des abzuprüfenden Wissens zu bestimmen (vgl. Tepner et al. 2012, 9; Krauss et al. 2011, 142f.). Die curricularen Inhalte für ein fundiertes rechnungswesenbezogenes Fachwissen unterscheiden sich aus schulischer und universitärer Perspektive (im Unterschied zur Mathematik) kaum (vgl. Bouley, in Vorb.). Die akademische Referenzdisziplin weist hinsichtlich ihrer Kanonisierung und Behandlung demnach analoge Strukturen zur Unterrichtspraxis im Rechnungswesen auf. Da zur adäquaten Vorbereitung des Unterrichts „Wissen erforderlich ist, das über die Schulstufe hinausgeht, in der unterrichtet wird" (Tepner et al. 2012, 10), wird im vorliegenden Forschungsvorhaben als Zielperspektive der Testkonstruktion ein profundes Verständnis schulischer Rechnungsweseninhalte angelegt.

\section{Fachdidaktisches Wissen}

Da das Fachwissen zwar insgesamt als eine notwendige, nicht aber als eine hinreichende Voraussetzung für die verständnisorientierte Gestaltung von 
Lerninhalten im Unterricht angesehen wird (vgl. Schlump 2010, 233), rückt das fachdidaktische Wissen in den Fokus der Testentwicklung. Dieses setzt Kenntnisse über rechnungswesenbezogene Fachinhalte voraus, stellt ,aber selbst ein besonderes unterrichts- und schülerbezogenes fachliches Wissen" dar (Baumert/Kunter 2011, 37).

Bei der Analyse einzelner Studien, die sich mit der Struktur der professionellen Kompetenz beschäftigen, lässt sich eine ganze Reihe unterschiedlicher Facetten des fachdidaktischen Wissens ausmachen. Allerdings zeigt sich, dass das Wissen über den Umgang mit Schülerfehlern und das Wissen über das Zugänglichmachen von Inhalten als gemeinsame "Schnittmenge” der Zusammenschau verschiedener fachdidaktischer Facetten angesehen werden kann (eine Übersicht über verschiedene Modellierungsmöglichkeiten findet sich u.a. bei van Driel/Verloop/de Vos 1998; Park/Oliver 2008). Darüber hinaus stellt die Auswahl von Aufgaben im Rechnungswesenunterricht, ähnlich wie im Mathematikunterricht, ein wesentliches Mittel zur Steuerung kognitiv anspruchsvoller Lernprozesse dar (vgl. Brunner et al. 2006). Daher wird das fachdidaktische Wissen im KoMeWP-Projekt anhand der drei Facetten (1) Schülerkognition, (2) Potenzial von Aufgaben und (3) Zugänglichmachen konzeptualisiert. Tabelle 1 bietet eine Übersicht über die Fassung der fokussierten Wissenskomponenten und -facetten.

Das Fachwissen und das fachdidaktische Wissen wird im Paper-PencilTest sowohl über geschlossene (Multiple Choice oder Complex Multiple Choice) als auch über offene Aufgaben erfasst. Im Bereich der Fachdidaktik werden zusätzlich „Situational Judgement”-Aufgaben (vgl. McDaniel/Nguyen 2001) entwickelt, um eine handlungsnahe Abfrage dieser Wissenskomponente zu ermöglichen. Im Testinstrument wird letztendlich ein Verhältnis von 2:1 (PCK:CK) angestrebt, um die Komponente des fachdidaktischen Wissens stärker zu betonen. Die Inhaltsbereiche, die den Fachdidaktikitems zugrunde liegen, werden jeweils zusätzlich in einem Fachwissensitem abgeprüft. Die reinen Fachwissensitems dienen somit der Kontrolle, da das fachliche Wissen als Voraussetzung für die Lösung fachdidaktischer Items gilt.

Bezogen auf das verankerte kognitive Anforderungsniveau (in Anlehnung an Anderson/Krathwohl 2001) decken die Items insgesamt ein breites Spektrum ab und reichen von der Abfrage einfacher Definitionen oder der Wiedergabe typischer Fehler (Reproduktion), über Buchungsaufgaben (Anwendung) bis hin zu Transfer-, Entwicklungs- und Beurteilungsaufgaben (Entwickeln/Bewerten). Die Probanden müssen dabei sowohl algorithmische Kenntnisse (System der Buchführung) als auch ökonomische Kenntnisse (Beschreibung der Auswirkungen verschiedener Buchungen) demonstrieren. 
Tab. 1: Definition der fachwissenschaftlichen und fachdidaktischen Facetten

\begin{tabular}{|c|c|}
\hline Komponenten & $\begin{array}{l}\text { Beschreibung der Facette } \\
\text { Beispielhafter Teilaspekt }{ }^{4}\end{array}$ \\
\hline $\begin{array}{l}\text { Fachdidaktik: Wissen } \\
\text { über } \\
\text { Schülerkognitionen }\end{array}$ & $\begin{array}{l}\text { Benennen und Erklären von (typischen) Schülerfehlern oder } \\
\text { Verständnisschwierigkeiten in einem Inhaltsbereich. } \\
\text { Beispiel: Der Lehramtsanwärter kann die Ursache für einen Feh- } \\
\text { ler in einer Schülerlösung analysieren. }\end{array}$ \\
\hline $\begin{array}{c}\text { Fachdidaktik: } \\
\text { Wissen über das Po- } \\
\text { tenzial von } \\
\text { Aufgaben }\end{array}$ & $\begin{array}{l}\text { Erkennen und Darlegen des kognitiven Aktivierungspotenzials } \\
\text { einer Aufgabe in einem Inhaltsbereich. } \\
\text { Beispiel: Der Lehramtsanwärter kann beurteilen, ob das Vorwis- } \\
\text { sen der Schüler für die Bearbeitung einer Rechnungswesenaufgabe } \\
\text { ausreichend ist. }\end{array}$ \\
\hline $\begin{array}{c}\text { Fachdidaktik: } \\
\text { Wissen über das Zu- } \\
\text { gänglichmachen von } \\
\text { Inhalten }\end{array}$ & $\begin{array}{l}\text { Erklären und Veranschaulichen eines Inhaltsbereichs anhand } \\
\text { verschiedener Zugangsmöglichkeiten. } \\
\text { Beispiel: Der Lehramtsanwärter kann multiple Erklärungen zu ei- } \\
\text { nem Sachverhalt generieren. }\end{array}$ \\
\hline Fachwissen & $\begin{array}{l}\text { Bearbeitung von fachlichen Fragen } \\
\text { Identifikation von Schülerfehlern } \\
\text { Beispiel: Der Lehramtsanwärter kann rechnungswesenbezogenen } \\
\text { Großen und Begriffe zueinander in Beziehung setzen. Der Lehr- } \\
\text { amtsanwärter kann Schülerfehler in Lösungen zu Buchführungs- } \\
\text { aufgaben aufdecken. }\end{array}$ \\
\hline
\end{tabular}

Die beiden in den nachstehenden Abbildungen 1 und 2 exemplarisch aufgeführten Testitems sollen die Operationalisierung der fachdidaktischen Facetten „Schülerkognitionen” und „Zugänglichmachen” illustrieren und beziehen sich auf die in Tabelle 1 dargestellten Teilaspekte.

Abb. 1: Testitem zum fachdidaktischen Wissen - Schülerkognitionen (,Anwenden”)

Sie wiederholen zu Beginn der Unterrichtsstunde die Begriffe Inventur, Inventar und Bilanz. Susi meldet sich und fragt: „Warum wird am Jahresende durch die Inventur nochmals alles gezählt, wenn doch schon Endbestände durch das Buchen vorliegen?"

a) Welchen wesentlichen Aspekt der Inventur übersieht Susi?

b) Vielen Schülern ist der Zusammenhang zwischen Inventur und Bilanz am Ende ihrer Schulzeit nicht klar. Woran könnte dies liegen?

4 Die Beispiele stellen nur eine Auswahl der Teilaspekte der Facetten dar. 
Abb. 2: Testitem zum fachdidaktischen Wissen - Zugänglichmachen (,Bewerten”)

Im Unterricht fragt Paul, wie er sich merken kann, auf welcher Seite Erträge und Aufwendungen bei Erfolgskonten gebucht werden.

Maria antwortet: „A wie Aufwand kommt im Alphabet vor E wie Ertrag, weswegen im Sinne einer Reihenfolge von links nach rechts, die Aufwendungen im Soll und die Erträge im Haben stehen."

a) Bitte bewerten Sie die Erklärung von Maria.

b) Bitte geben Sie Paul eine alternative Erklärung.

Nach dieser theoretischen Konzeptualisierung des professionellen Wissens für das KoMeWP-Testinstrument wird im Folgenden die inhaltliche Dimension der Testitems definiert.

\section{Die inhaltliche Dimension der Testkonstruktion}

Das Unterrichtsfach Rechnungswesen gliedert sich traditionell in die Teilbereiche „Inventur und Bilanz”, „doppelte Buchführung” sowie „Kosten-Leistungsrechnung" (Sloane 1996, S. 26). Diese Teilaspekte bestimmen die Fachstruktur der beruflichen Vollzeitschulen und bleiben, trotz Einführung des Lernfeldkonzepts, auch im Berufsschulunterricht erhalten. Während Inventar und Bilanz meist als Einführung in das Rechnungswesen betrachtet werden und die Kosten-Leistungsrechnung als zusätzliches, nachgelagertes Thema angesehen wird, steht die doppelte Buchführung im Mittelpunkt des Unterrichts (vgl. ebd., S. 26). Auf Grund dieser Tatsache soll die „Buchführung” auch bei der Entwicklung des vorliegenden Testinstruments einen zentralen Stellenwert einnehmen.

\section{Analyse der (Rahmen-) Lehrpläne und Schulbücher}

Um den verschiedenen späteren Einsatzbereichen angehender Lehrkräfte bei der inhaltlichen Ausrichtung des Testinstruments gerecht zu werden, wurden (Rahmen-)Lehrpläne und einschlägige Schulbücher im Hinblick auf die dort beschriebenen Buchführungsinhalte analysiert. Zielsetzung war es, ein Rahmenmodell aufzuspannen, das die „Schlüsselinhalte” des Lerninhaltsbereichs abdeckt.

In diesem Zusammenhang wurden (Rahmen-)Lehrpläne ausgewählter Ausbildungsberufe und Vollzeitschulen aller Bundesländer sowie verschiedene Schulbücher analysiert. Bei den Lehrplänen der Vollzeitschulen wurden jene berücksichtigt, die in (fast) allen Bundesländern vertreten sind (Berufsfachschule Wirtschaft und Wirtschaftsgymnasium). Bei den Rahmenlehr- 
plänen wurden die ausbildungsstärksten herausgegriffen (Einzelhandelskaufleute, Industriekaufleute, Bürokaufleute sowie Groß- und Außenhandelskaufleute). Die Analyse der Schulbuchinhalte gründete dagegen auf sieben (auflagenstarken) Fachbüchern aus dem Bereich des (externen) Rechnungswesens.

Das methodische Vorgehen bei der (Rahmen-)Lehrplan- sowie Schulbuchanalyse orientierte sich an der Systematik einer offenen Inhaltsanalyse. Die curricular verankerten Buchführungsinhalte wurden durch die Bildung von Kategorien systematisiert. Ziel war es hierbei, einen möglichst vollständigen Überblick über die in den Dokumenten abgebildeten Inhaltsbereiche zu erhalten. Die Kategorien wurden dann wiederum in übergeordneten Lerninhaltsbereichen zusammengefasst und systematisiert.

Obwohl die Dokumente nicht durchgehend vergleichbar waren (u.a. aufgrund großer Unterschiede hinsichtlich des Detaillierungsgrades der Lehrpläne), führten die Analysen u.E. zur Isolation der wesentlichen Grundkenntnisse und -prozeduren der betrieblichen Buchführung. Diese lassen sich in die fünf nachfolgenden Lerninhaltsbereiche gliedern: (1) Aufgaben, Bedeutung und Rechtgrundlagen, (2) System der Doppik, (3) System der Umsatzsteuer, (4) Beschaffungs- und Absatzprozesse und (5) Jahresabschluss.

Der Lerninhaltsbereich (1) fokussiert die wesentlichen Grundkenntnisse der Buchführung und umfasst neben rechtlichen Grundlagen auch die wesentlichen Fachtermini (wie Inventur, Inventar und Bilanz), die für ein grundlegendes Verständnis notwendig sind. Die Lerninhaltsbereiche (2) und (3) beziehen sich hingegen auf die Grundprozeduren, wie der Unterscheidung und Buchung von Bestands- und Erfolgsvorgängen sowie der zugrunde gelegten Wertschöpfung im betrieblichen Leistungsprozess. Es sollen die Grundbausteine der Buchungstechnik näher gebracht werden, die in den weiteren Bereichen der Buchführung (wie Beschaffungs- und Absatzprozesse sowie Personal-, Anlagen- und Finanzwirtschaft als Nebenfunktionen) zum Tragen kommen. Es erfolgt des Weiteren mit Lerninhaltsbereich (4) eine Fokussierung auf die Primärfunktionen des betrieblichen Leistungsprozesses. Die Berücksichtigung des Jahresabschlusses als Lerninhaltsbereich (5) rundet die Inhaltsauswahl ab, da dieser die Grundlage für ein umfassendes, gesamtzusammenhängendes ökonomisches Verständnis der Bedeutung der Buchführung darstellt (vgl. Preiß 2005, 71).

Bezogen auf die oben beschriebenen Inhaltsbereiche stehen verschiedene Möglichkeiten der didaktischen Sequenzierung sowie der methodischen Umsetzung zur Verfügung. Im schulischen und universitären Rechnungswesen dominiert bislang die Bilanzmethode (vgl. Seifried 2004, 29). Dies spiegelt sich auch in den oben beschriebenen Analysen von (Rahmen-)Lehrplänen und Schulbüchern wider. Zentrales Charakteristikum der Bilanzmethode ist die Auflösung des gesamten Buchhaltungsstoffes in methodische Einheiten, wobei die Bilanz den „Ausgangs-, Mittel- und Zielpunkt aller Buchungsar- 
beiten" bildet (vgl. Butze 1936, S. 46f. zit. n. Seifried 2004, 29). Auch wenn das bilanzmethodische Vorgehen einen starken Einfluss auf die systematische Vermittlung des Rechnungswesens in Schule und Hochschule besitzt, soll die an dieser Methode geübte Kritik (vgl. Preiß 2000) im Rahmen der Testkonstruktion nicht unberücksichtigt bleiben. Deshalb werden, insbesondere in den Items zum fachdidaktischen Wissen, gezielt Schwächen und Kritikpunkte des traditionellen Rechnungswesenunterrichts sowie fachdidaktische Alternativen (Wirtschaftsinstrumentelles Rechnungswesen) thematisiert.

\section{Analyse von rechnungswesenbezogenen Fehlern und \\ Verständnisschwierigkeiten}

Die Konzeptualisierung der fachdidaktischen Facetten "Schülerkognitionen” und „Zugänglichmachen” sowie „Aufgabenpotential” zeigt, dass sich diese oftmals auf rechnungswesenbezogene Fehler und Verständnis- bzw. Bearbeitungsschwierigkeiten beziehen (siehe beispielhaft Tab. 1). Es war deshalb naheliegend, eine weitergehende Analyse anzuschließen, die eine Identifikation einschlägiger, realistischer Fehler und Verständnisschwierigkeiten innerhalb der oben genannten fünf Lerninhaltsbereiche des Rechnungswesens erlaubt.

Hierzu konnte auf bestehende Interviewdaten aus einem bereits abgeschlossenen Projekt der Forschergruppe um Seifried und Wuttke (vgl. Wuttke/Seifried 2012; Türling et al. 2011) zurückgegriffen werden. Durch eine Reanalyse der 2009 erhobenen Interviewdaten von 51 Lehrkräften an berufsbildenden Schulen konnten Informationen $\mathrm{zu}$ rechnungswesenbezogenen Verständnisschwierigkeiten und typischen Fehlern gewonnen werden. Die von den Lehrkräften genannten Schülerfehler wurden mit Hilfe der Software MAXQDA kodiert und dem entsprechenden Lerninhaltsbereich zugeordnet. Im Zentrum stand die qualitative Auflistung sämtlicher Fehler und Verständnisschwierigkeiten in den Äußerungen der Probanden. Die obigen Analyseergebnisse wurden mit den Befunden anderer Studien abgeglichen (vgl. Pawlik 1980; Seifried 2004; Tramm/Hinrichs/Langenheim 1996) und entsprechend ergänzt. Auf dieser Grundlage konnten insgesamt 80 Einzelaspekte identifiziert werden, die sich auf Verständnisschwierigkeiten und Fehler im Unterricht beziehen und unmittelbare Anknüpfungspunkte für die Konstruktion realitätsnaher Testitems bieten. Nachfolgend werden exemplarische Einzelaspekte dargestellt:

- Erkennen, dass die Bilanz durch die Inventur aufgestellt wird und die Buchungen nur die Sollwerte darstellen (Aufgaben, Bedeutung und Rechtsgrundlagen).

- Erkennen, ob ein Aktiv- oder Passivkonto vorliegt (System der Doppik).

- Verstehen, dass die Umsatzsteuer einen durchlaufenden Posten darstellt (System der Umsatzsteuer). 
- Erkennen der jeweiligen Perspektive: Käufer oder Verkäufer (Beschaffungs- und Absatzprozesse).

- Interpretation der Auswirkungen des Unternehmensergebnisses (Jahresabschluss).

Abschließend lassen sich die einzelnen Vorarbeiten in nachfolgender Abbildung 3 veranschaulichen. Es wird ersichtlich, dass ein jedes Testitem durch die Wissenskomponente bzw. -facette, den Lerninhaltsbereich sowie dem kognitiven Anforderungsniveau bestimmbar wird.

Abb. 3: Rahmenmodell zur Konstruktion von Testitems zur Erfasssung des fachlichen und fachidaktischen Wissens. Das Fachwissen ist gestrichelt dargestellt. ${ }^{5}$

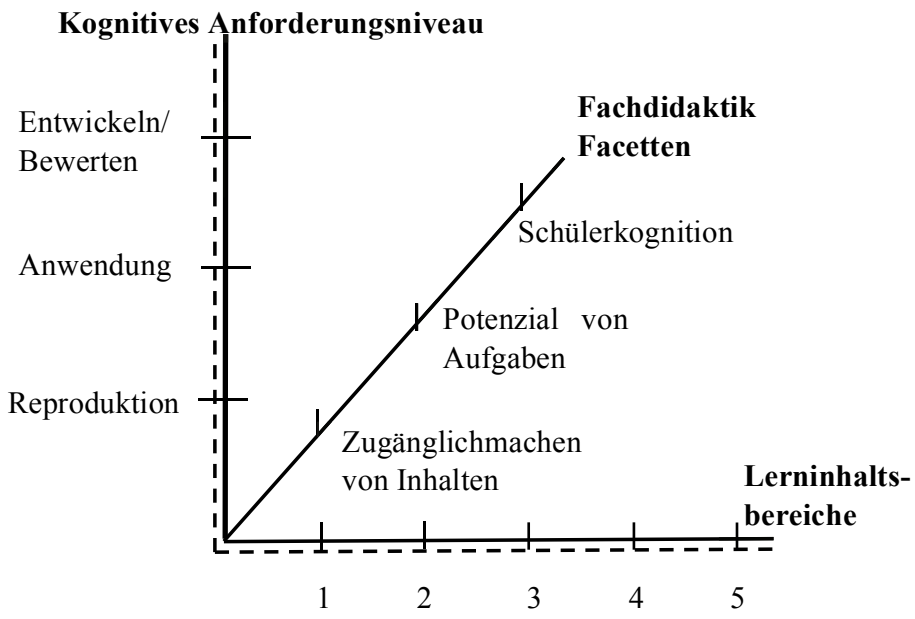

\section{Fazit und Ausblick auf nächste Arbeitsschritte}

Der vorliegende Beitrag erläutert die Zielsetzung des Projekts KoMeWP und gibt einen Überblick über die Vorarbeiten für die Erstellung eines PaperPencil-Testinstruments zur Erfassung des professionellen Wissens angehender Wirtschaftspädagogen. Ausgehend von der theoretischen Konzeptualisierung der Komponenten Fachwissen und fachdidaktisches Wissen wird die

$5 \quad 1=$ Aufgaben, Bedeutung \& Rechtsgrundlagen; 2 = System der Doppik; 3 = System der Umsatzsteuer; 4 = Beschaffungs- \& Absatzprozesse; 5 = Jahersabschluss. 
Inhaltsauswahl für die Testitems begründet. Durch die beschriebenen Vorarbeiten wurde eine Basis zur Entwicklung realistischer und kritischer Aufgaben geschaffen, die angehende Lehrkräfte in ihrem Arbeitsalltag bewältigen müssen. Im Zuge einer Pretestung im Frühjahr 2013 wird der gesamte Itempool erprobt und durch Fachexperten beurteilt. Auf Basis der sich hieraus ergebenden Befunde wird das Testinstrument erstellt, welches im Herbst 2013 in einer deutschlandweiten Querschnittsstudie zur Erfassung des fachdidaktischen und fachlichen Wissens angehender Wirtschaftspädagogen zum Einsatz kommen wird. Die Validierung des Tests wird durch eine zusätzliche Erhebung an vier Universitätsstandorten in Österreich gesichert.

\section{Literatur}

Anderson, L.W./Krathwohl, D. R. (2001): A Taxonomy for Learning, Teaching, and Assessing. A Revision of Bloom's Taxonomy of Educational Objectives. New York.

Baumert, J./Kunter, M. (2011): Das Kompetenzmodell von COACTIV. In: Kunter, M./Baumert, J./Blum, W./Klusmann, U./Krauss, S./Neubrand, M. (2011): Professionelle Kompetenz von Lehrkräften - Ergebnisse des Forschungsprogramms von COACTIV. Münster: Waxmann, S. 29-53.

Blömeke, S./Zlatkin-Troitschanskaia, O. (2013): Forschungsprojekte im Rahmen von KoKoHs. Online: http://www.kompetenzen-im-hochschulsektor.de/174_DEU_ HTML.php (18-03-2013).

Blömeke, S./Zlatkin-Troitschanskaia, O./Kuhn, C./Fege, J. (2013): Modeling and Measuring Competencies in Higher Education. Task and Challenges. Rotterdam: Sense Publishers.

Blömeke, S./Bremerich-Vos, A./Haudeck, H./Kaiser, G./Nold, G./Schwippert, K./ Willenberg, H. (2011): Kompetenzen von Lehramtsstudierenden in gering strukturierten Domänen - Erste Ergebnisse aus TEDS-LT. Münster: Waxmann, S. 724.

Bouley, F. (in Vorb.): Analyse der Lerngelegenheiten im Studium der Wirtschaftspädagogik. Unveröffentl. Masterarbeit an der Goethe-Universität Frankfurt a.M. (Prof. Dr. E. Wuttke).

Brunner, M./Kunter, M./Krauss, S./Klusmann, U./Baumert, J./Blum, W./Neubrand, M./Dubberke, T./Jordan, A./Löwen, K./Tsai, Y. M. (2006): Die professionelle Kompetenz von Mathematiklehrkräften: Konzeptionalisierung, Erfassung und Bedeutung für den Unterricht. Eine Zwischenbilanz des COACTIV-Projekts. In: Prenzel, M./Allolio-Näcke, L. (Hrsg.): Untersuchungen zur Bildungsqualität von Schule. Abschlussbericht des DFG-Schwerpunktprogramms. Münster: Waxmann, S. 54-82.

Butze, F. (1936): Der Wert der Buchhaltung als Bildungsgut der wirtschaftsberuflichen Schulung. Dissertation: Handelshochschule Leipzig. 
Gruber, H./Mandl, H. (1996): Das Entstehen von Expertise. In: Hoffmann, J./Kintsch, W. (Hrsg.): Enzyklopädie der Psychologie, Theorie und Forschung, Kognition. Bd. 7 Lernen. Göttingen: Hogrefe, S. 583-615.

Krauss, S./Blum, W./Brunner, M./Neubrand, M./Baumert, J./Kunter, M./Besser, M./ Elsner, J. (2011): Konzeptualisierung und Testkonstruktion zum fachbezogenen Professionswissen von Mathematiklehrkräften. In: Kunter, M./Baumert, J./Blum, W./Klusmann, U./Krauss, S./Neubrand, M. (Hrsg.): Professionelle Kompetenz von Lehrkräften. Ergebnisse des Forschungsprogramms COACTIV. Münster: Waxmann Verlag, S. 135-161.

Kuhn, C./Zlatkin-Troitschanskaia, O. (2011): Assessment of competencies among university students and graduates - Analyzing the state of research and perspectives. Johannes Gutenberg University Mainz: Arbeitspapiere Wirtschaftspädagogik, 59.

Kunter, M./Baumert, J./Blum, W./Klusmann, U./Krauss, S./Neubrand, M. (2011): Professionelle Kompetenz von Lehrkräften - Ergebnisse des Forschungsprogramms von COACTIV. Münster: Waxmann.

McDaniel, M.A./Nguyen, N.T. (2001): Situational Judgement Test. A Review of Practice and Construct Assessed. International Journal of Selection and Assessment, 9,1/2, pp. 103-113.

Park, S./Oliver, S. J. (2008): Revisiting the Conceptualisation of Pedagogical Content Knowledge (PCK): PCK as a Conceptual Tool to Understand Teachers as Professionals. Research in Science Education, 38(3), pp. 261-284.

Pawlik, W. (1980): Fachdidaktik des Unterrichts in Rechnungswesen. In: Zeitschrift für Berufs- und Wirtschaftspädagogik, 76(1), S. 33-43.

Preiß, P. (2000): Der Rechnungswesenunterricht als Beitrag zum Verständnis ökonomischer Zusammenhänge und wirtschaftlicher Entscheidungen. In: Bundesverband der Lehrer an Wirtschaftsschulen (Hrsg.): Funktionswandel des Rechnungswesens: Von der Dokumentation zur Steuerung, Heft 44 der Sonderschriftreihen des VLW, S. 7-29.

Preiß, P. (2005): Entwurf eines Kompetenzkonzepts für den Inhaltsbereich Rechnungswesen/Controlling. In: Gonon, P./Klauser, F./Nickolaus, R./Huisinga, R. (Hrsg.): Kompetenz, Kognition und neue Konzepte der beruflichen Bildung. Wiesbaden: VS Verlag für Sozialwissenschaften, S. 67-84.

Seifried, J. (2004): Fachdidaktische Variationen in einer selbstorganisationsoffenen Lernumgebung. Wiesbaden: Deutscher Universitäts-Verlag.

Seifried, J./Wuttke, E. (2010): Professionelle Fehlerkompetenz - Operationalisierung einer vernachlässigten Kompetenzfacette von (angehenden) Lehrkräften. Wirtschaftspsychologie, 12(4), S. 17-28.

Seifried, J./Türling, J. M./Wuttke, E. (2010): Professionelles Lehrerhandeln - Schülerfehler erkennen und für Lernprozesse nutzen. In: Warwas, J./Sembill, D. (Hrsg.): Schulleitung zwischen Effizienzkriterien und Sinnfragen. Baltmannsweiler: Schneider Verlag Hohengehren, S. 137-156.

Seifried, J./Wuttke, E./Schmitz, B. (2011): Modellierung und Erfassung fachwissenschaftlicher und fachdidaktischer Kompetenzen im wirtschaftspädagogischen Studium - Projektskizze (Verbundprojekt). Unveröffentlichter Förderantrag an das Bundesministerium für Bildung und Forschung.

Sloane, P. F. E. (1996): Didaktik des Rechnungswesens. Wirtschaftspädagogisches Forum. Pfaffenweiler: Centaurus. 
Schlump, S. (2010): Kompetenzen von Lehrpersonen zur Konstruktion von Lernaufgaben. In: Kiper, H/Meints, W./Peters, S./Schlump, S. (Hrsg.): Lernaufgaben und Lernmaterialen im kompetenzorientierten Unterricht. Stuttgart: Kohlhammer Verlag, S. 224-236.

Shulman, L. (1986): Those who understand. Knowledge growth in teaching. Educational Researcher, 15(2), pp. 4-14.

Tepner, O./Borowksi, A./Dollny, S./Fischer, H. E./Jüttner, M./Kirschner, S./Leutner, D./Neuhaus, B. J./Sandmann, A./Sumfleth, E./Thillmann, H./Wirth, J. (2012): Modell zur Entwicklung von Testitems zur Erfassung des Professionswissens von Lehrkräften in den Naturwissenschaften. Zeitschrift für Didaktik der Naturwissenschaften, 18, S. 7-28.

Tramm, T./Hinrichs, K./Langenheim, H. (1996): Lernschwierigkeiten im Buchführungsunterricht. In: Preiß, P./Tramm, T. (Hrsg.): Rechnungswesenunterricht und ökonomisches Denken. Wiesbaden: Gabler, S. 158-221.

Türling, J. M./Seifried, J./Wuttke, E./Gewiese, A./Kästner, R. (2011): ,Typische' Schülerfehler im Rechnungswesenunterricht - Befunde einer Interviewstudie. Zeitschrift für Berufs- und Wirtschaftspädagogik, 107 (3), S. 390-407.

Van Driel, J. H./Verloop, N./de Vos, W. (1998): Developing Science Teachers' Pedagogical Content Knowledge. Journal of Research in Science Teaching, 35(6), pp. 673-695.

Weinert, F. E. (2001): Vergleichende Leistungsmessung in Schulen - eine umstrittene Selbstverständlichkeit. In: Weinert, F. E. (Hrsg.): Leistungsmessung in Schulen. 2. Aufl.. Weinheim und Basel: Beltz, S. 17-31.

Wuttke, E./Seifried, J. (2012): Ansätze der Identifikation typischer Schülerfehler - Ergebnisse aus Studien in kaufmännischen Schulen. Unterrichtswissenschaften, 40(2), S. 174-192.

Zlatkin-Troitschanskaia, O./Breuer, K. (2013): WiWiKom. Design und Instrumente. Online: http://www.wiwi-kompetenz.de/28.php (18-03-2013). 